\title{
Simulation of gait asymmetry and energy transfer efficiency between unilateral and bilateral amputees
}

\author{
Navid Aslani ${ }^{1} \cdot$ Siamak Noroozi $^{1} \cdot{\text { Khoo Shin } \mathrm{Yee}^{2,3} \cdot \text { Alex Ong Zhi Chao }}^{2,3}$. \\ Christine Maggs ${ }^{1}$
}

Published online: 11 April 2016

(c) The Author(s) 2016. This article is published with open access at Springerlink.com

\begin{abstract}
Efficient walking or running requires symmetrical gait. Gait symmetry is one of the key factors in efficient human dynamics, kinematics and kinetics. The desire of individuals with a lower-limb amputation to participate in sports has resulted in the development of energy-storingand-returning (ESR) feet. This paper analyses a case study to show the effect of symmetry and asymmetry as well as energy transfer efficiency during periodic jumping between simulated bilateral and unilateral runners. A custom gait analysis system is developed as part of this project to track the motion of the body of a physically active subject during a set of predefined motions. Stance and aerial times are accurately measured using a high speed camera. Gait frequency, the level of symmetry and the non-uniform displacement between left and right foot and their effects on
\end{abstract}

Navid Aslani

naslani@bournemouth.ac.uk

Siamak Noroozi

snoroozi@bournemouth.ac.uk

Khoo Shin Yee

mikeson.khoo@yahoo.com

Alex Ong Zhi Chao

zhichao83@gmail.com

Christine Maggs

cmaggs@bournemouth.ac.uk

1 Department of Design and Engineering, Faculty of Science and Technology, Bournemouth University, Talbot Campus, Fern Barrow, Poole, Dorset BH12 5BB, UK

2 Department of Mechanical Engineering, Engineering Faculty, University of Malaya, 50603 Kuala Lumpur, Malaysia

3 Advanced Shock and Vibration Research Group, Applied Vibration Laboratory, Block R, Faculty of Engineering, University of Malaya, Kuala Lumpur, Malaysia the position of the Centre of Mass (CM) were used as criteria to calculate both peak energies and transformation efficiency. Gait asymmetry and discrepancy of energy transfer efficiency between the intact foot and the ESR are observed. It is concluded that unilateral runners require excessive effort to compensate for lack of symmetry as well as asymmetry in energy transfer, causing fatigue which could be a reason why bilateral amputee runners using ESR feet have a superior advantage over unilateral amputees.

Keywords Gait symmetry - Kinetics - Unilateral and bilateral $\cdot$ Image processing

\section{Introduction}

Paralympic running/sprinting has experienced many technological changes. In competitive parasports, amputee's performance is the most important factor, above other prosthesis design considerations such as comfort and cost. The desire of individuals with a lower-limb amputation to participate in sports and the high demands of athletics have resulted in the development of energy-storing-and-returning (ESR) feet, capable of absorbing and returning energy in the same manner as a trampoline. Although ESR has been in service since 1985 , there is little theoretical or analytical treatment of the science behind the concept.

The energy efficiency of ESR prostheses can vary. Energy return rates have ranged between $31 \%$ [1] and $95 \%[2,3]$. Studies of the biological lower limb have shown that the ankle is able to generate a $241 \%$ energy return [1], making a prosthesis a restorative device with performance falling a long way short of that of a biological limb [4]. In a review of transtibial ESR prosthetic devices 
Hafner et al. [5] noted that lack of power generation is a major barrier in creating gait symmetry. However, there are advantages compared to a normal foot: (a) it does not suffer from fatigue; (b) it has fixed properties/characteristics allowing simple transformation of potential energy to kinetic energy to stored energy and back. This allows the energy to be stored in the body mass and not in the foot.

Limb kinematics in transtibial amputee subjects are similar to those for individuals with sound limbs while kinematics for transfemoral amputees show a large gait asymmetry between contralateral limbs [6]. Physiological function is similar between amputee and intact limb subjects while mechanical function in sprinting differs between these two categories [7]. ESR prostheses allow amputees to reach the same energy cost when running as able-bodied persons [2]. Brüggemann et al. [3] showed that lower inertial properties of a prosthesis result in less mechanical work in lifting and accelerating the legs. Hobara et al. [8] discussed some advantages of ESR such as shorter swing time and longer contact lengths and disadvantages such as lower ground reaction force [9] and higher injury risks.

Until now ESR feet have mostly been prescribed based on their static characteristics and linear static stiffness determined experimentally using idealised boundary conditions. Their dynamic characteristics are poorly understood and the limited literature available shows inadequate understanding of the science behind the vibration or harmonic response of the system. There are few studies of the dynamics characteristics of ESR, as discussed below.

Noroozi et al. [4], studied the dynamic characteristics of Elite Blade composite feet, demonstrating the performance enhancing characteristics of these feet when used by a bilateral amputee in the latter stages of longer distance races such as 200 and $400 \mathrm{~m}$. Vinney et al. [10] showed mathematical/FEA modelling of the ESR foot as means of performing inverse dynamics to be used to pre-set or tune the foot for walking, jogging, running and sprinting. Noroozi et al. [11] showed that modal analysis of the mass and foot system clearly determined the three main parameters in dynamic response: frequency, mode shape and damping. Noroozi et al. [12] also developed the basic theories behind the dynamic elastic response of these feet to cyclic and impulse excitation and also demonstrated their response to impulse synchronisation, which can result in a trampoline effect.

Current technology is not ready to provide perfectly symmetric gait for unilateral amputees. The difference between unilateral and bilateral dynamics when using ESR feet needs to be investigated further to determine participation of amputee athletes on both ethical [13] and physiological considerations [3, 7]. Further concerns have been raised about how this technology will be judged in the future to ensure ethical inclusion of such technology in disability sports [14]. This is also substantiated by Hassani et al. [15], who showed statistically that bilateral amputee using ESR feet dominated the Paralympic Games post 2008, when these ESR feet were first introduced.

\subsection{Symmetry}

By definition, asymmetry, or lack of symmetry, appears to be a relevant for differentiating normal from pathological gaits. Gait asymmetry is often described as a ratio of the kinematic or kinetic parameters between the right and left sides [16]. Different parameters have been used to determine gait asymmetry for amputees such as ratio index [17], coefficients of variation [18], correlation coefficients [19], symmetry index [20], variance ratios [21], principal component analysis [22], and root-mean-square difference [23], symmetry angle [24], butterfly plot [25] and autocorrelation coefficient [26]. According to Kaufman et al. [16], most of these tools have major limitations because they do not provide a measurement of the symmetry magnitude. Hence, the asymmetry effect cannot be quantified. This study proposes to use the jumping frequency and gait efficiency associated with displacement of centre of mass (CM) to investigate and quantify the asymmetry effect between the bilateral and unilateral amputees.

\subsection{Energy transfer}

It is known that energetic costs of locomotion depend largely on body size, gait and speed. For example, previous research has shown that the energetic cost of walking and running increases progressively with the speed of movement [27]. Differences in energetic cost for different gait patterns such as running and walking have been also demonstrated [28, 29]. Energy consumption is commonly measured using heart rate and oxygen consumption, which can be further used in the analysis of the percent MHR (Maximum Heart Rate) and gait efficiency. However, it is difficult with these methods to detect the change due to large variability and therefore only suitable to be used as indicator of overall effort. Hall [30] and Rocha-Vieira [31] showed that the oscillation of the CM on the vertical plane is higher when running compared to walking, leading to a higher energy expenditure. A detailed discussion about the mechanical energy consequences for changes of the $\mathrm{CM}$ at different gravity values and walking speeds can be found in Cavagna et al. [32]. CM displacement is proportional to the energy expenditure of the person while jumping according to previous studies. The gait energy transfer efficiency for jumping activities is equal to displacement of CM during the aerial phase (output mechanical energy) divided by CM displacement at stance phase (input stored energy). In this 
study, the vertical oscillation of CM is used to evaluate the energy transfer efficiency between the simulated bilateral and unilateral amputees and the penalty in energy consumption needed to overcome the lack of gait symmetry.

Recent studies in this field have failed to propose a better model or improved understanding of the science involved. The effect of various parameters on the overall outcome is still undetermined. Therefore the gaps in the technology needed to achieve symmetric gait in in all classes of amputees still exists.

This paper simulates unilateral and bilateral jumping condition to investigate gait symmetry and energy transfer efficiency.

\section{Methods}

Ethical approval for the study was obtained from the Bournemouth University Research and Knowledge Exchange Office. The participant gave his written informed consent before inclusion in the study.

\subsection{Data collection}

Experimentally, a series of LED markers were designed and developed by the research team and attached to the individual's leg: two markers on the foot, two between the

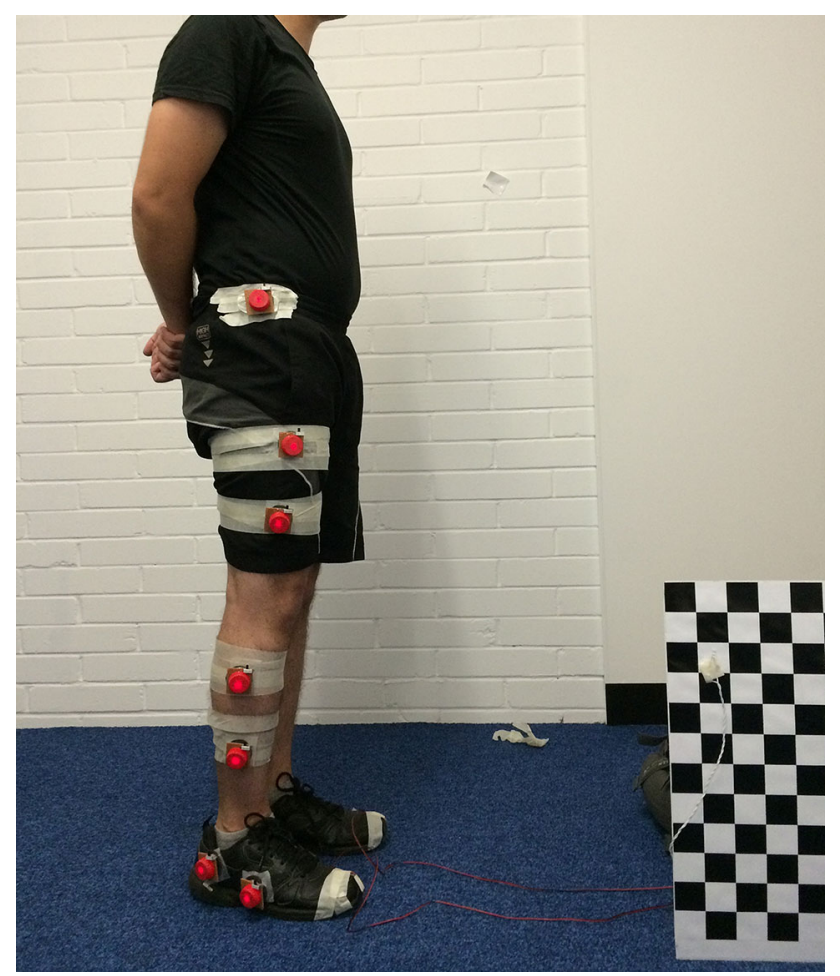

Fig. 1 Experiment setup ankle and knee, two between the knee and hip joint and one on the iliac tubercle as shown in Fig. 1. These markers are designed to emit light and be tracked using a high-speed camera (120 frames per second).

The camera is calibrated in a $2 \mathrm{D}$ plane using a custom checkerboard pattern measuring intrinsic and extrinsic camera parameters to compensate for the effect of lens distortion as well as camera translation and rotation. The participant was asked to jump along this $2 \mathrm{D}$ calibrated plane. Using a calibrated screen one can measure both absolute and relative displacement and rotation of every element of the leg and the CM. Although the absolute position of the CM is not measured in this study, its vertical displacement is determined from the vertical movement of the iliac tubercle.

The test is performed inside a darkened room to compensate for the effects of optical disturbance while processing motion. A custom code in MATLAB (Mathworks, USA) was developed using the image processing toolbox. The code automatically detects links between foot, tibia and femur and their relative position and angular rotation, while displacement of the marker mounted on the Iliac crest is considered as displacement of $\mathrm{CM}$ of the person in a 2D plane. The MATLAB code then converts pixel coordinates into units of measurement $(\mathrm{mm})$.

Calibration accuracy is evaluated by performing known motion of markers in different directions in calibrated 2D plane and maximum error of $2 \mathrm{~mm}$ was observed.

A custom optical trigger is made using Force Sensing Resistor (FSR) mounted below the individual's shoes. Stance phase is detected while individual's foot touches the ground, when the trigger LED turns ON. The ON trigger LED is recognized in the image processing code and frames with ON trigger LED are recorded as stance phase.

\subsection{Subject}

One physically active subject (age 29 years; body weight $67 \mathrm{~kg}$ ) volunteered to participate in the study. The subject was experienced in jumping on a trampoline and was informed of the purpose of the study. The subject gave written informed consent to participate in the study. The subject performed consecutive bouncing jumps alternating left and right legs while keeping his hands interlocked behind his back. He was instructed to reach maximum jumping height similarly in all jumps while keeping the jumping frequency as constant as possible within each test. A number of practice trials were performed before the measured trial.

\subsection{Experimental protocol}

Three different experiment settings were used. 
(a) Jumping up and down along $Y$ axis with both feet contacting the ground (Fig. 2a).

(b) Jumping up and down along $Y$ axis both feet contacting the small trampoline (Fig. 2b).

Movement of half of the trampoline was constrained by placing a rigid wooden box below one side of it, having the same height of trampoline. In this way one side of the trampoline (below one leg) acts like a rigid floor surface (known as the ground) while the other side (below the other leg) maintain its spring/elastic effect. The box was adjusted to minimise the leg length discrepancy during stance. If one foot bounces on the ground and one on trampoline, it will result in excessive use of energy to compensate for the lack of symmetry in energy consumption. Therefore, the effect of one of the key parameters behind difference in performance between unilateral and bilateral amputees in longer distance races could be investigated. (c) Jumping up and down along the $Y$ axis while one foot contacts the rigid half of trampoline (ground) and the other foot contact the free half of trampoline (Fig. 2c).

The developed MATLAB code allowed the markers to be tracked on the image allowing a full kinematic study as well as position, velocity and acceleration monitoring of the individual's leg and CM. The design of this test is informed by the Simulink (Mathworks, USA) simulation of the same setup allowing systematic assessment of the dynamics based on parameters affecting the gait.

For all three experimental settings joint kinematics for both sides are plotted and a symmetric gait pattern is observed between right and left legs. It is also observed that iliac tubercle moves only vertically without rotation either forward or backward for both legs. Hence it is assumed the change of $\mathrm{CM}$ caused by joint rotation is similar for left and right legs, so the effect of energy

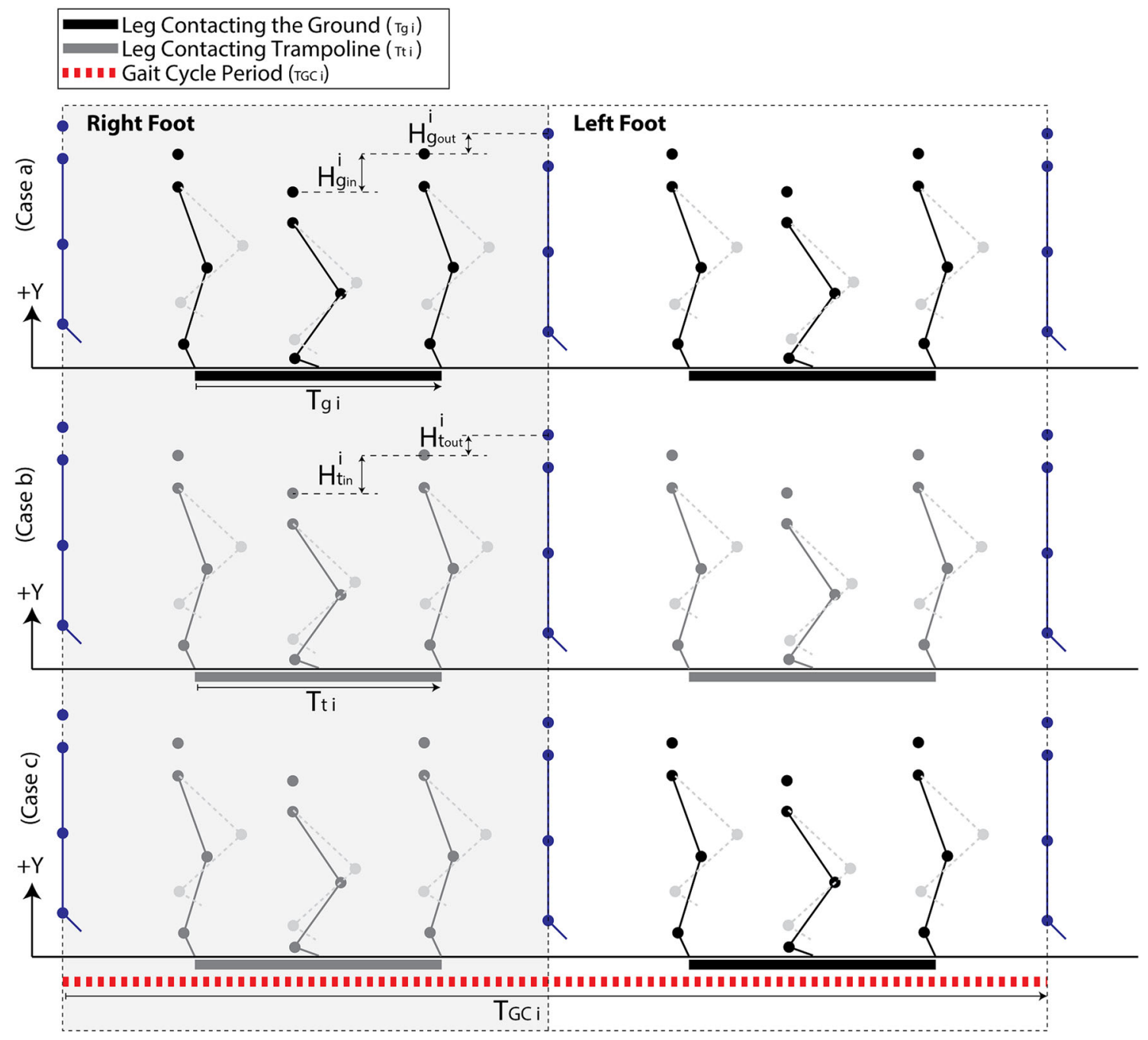

Fig. 2 Test setups. a Both feet on ground, b both feet on trampoline, $\mathbf{c}$ one foot on the ground the other on the trampoline 
storage and return on $\mathrm{CM}$ is compared between right and left.

\section{Results}

In the first test (Fig. 2a), the test subject jumps up with his right leg touching the ground. When the shoe contacts the ground, trigger LED turns $\mathrm{ON}$ and stance phase is automatically detected by image processing code. The moment his right foot leaves the ground (entering flight phase) aerial phase is detected having trigger LED OFF. Aerial phase continues till he reaches his maximum flight height and while going down this time his left foot touches the ground entering stance phase detected by ON trigger LED. Displacement of CM in time and stance and aerial phases are shown in (Fig. 3). Test two (Fig. 2b) was performed following the same sequence as test one, this time with both legs bouncing on the trampoline. Displacement of CM in time, stance, and aerial phases are shown (Fig. 4).

As shown in Table 1 for the case where both feet contact the ground (Case 1) the Jump frequencies $\left(f_{g 1}, f_{g 2}, f_{g 3}, f_{g 4}\right)$ are identical $\left(f_{\text {average }}=2.52 \mathrm{~Hz}\right.$ ). The same observation is found in the case where both feet contact the trampoline (Case 2) where the Jump frequencies $\left(f_{t 1}, f_{t 2}, f_{t 3}, f_{t 4}\right)$ are identical $\left(f_{\text {average }}=1.95 \mathrm{~Hz}\right.$ ) but different from Case 1 .

In the third test (Fig. 2c), the individual jumps up with his right foot touching the ground; the moment his right foot leaves the ground contact he enters aerial phase till he reaches his maximum height and while going down this time his left foot bounces on the trampoline in stance phase. Displacement of CM in time and stance and aerial phases are shown (Fig. 5).

As shown in Table 1, the subject found it hard to achieve the same jumping frequency when the average jump frequency while contacting the free half of trampoline,$\frac{f_{t 1}+f_{t 2}}{2}=1.94 \mathrm{~Hz}$ was not identical to the average jump frequency while contacting the ground (rigid side of trampoline), $\quad \frac{f_{g 1}+f_{g 2}}{2}=2.43 \mathrm{~Hz}$. Therefore the person requires additional effort to achieve the desired constant jumping frequency between right and left legs.

The results indicated that an able-bodied person and bilateral amputee require minimum effort to keep jumping frequency constant (i.e., the case where both feet contacting the ground/trampoline) compare to the unilateral amputee (i.e., the case where one foot contacting the ground and another foot contacting the trampoline).

In addition, as shown in (Fig. 5) it is discovered that the gait cycle has the same period for all the cases $\left(T_{G C 1}=T_{G C 2}\right)$. Gait cycle describes the interval time/period where the same foot contacting to the ground before flight phase and contact to the ground during landing stage.

\subsection{Energy transfer efficiency}

The symmetry between right and left in jumping is found for the first two cases (Fig. 3) and (Fig. 4) where the CM displacements are almost constant while jump asymmetry occurs in the third case (Fig. 5) where CM displacements differ between the right and left foot. It may need further explanation to relate jump asymmetry and the CM displacement. So far, from the literature, CM displacement has been associated with energy expenditure/energy consumption rate but not gait asymmetry.

In jumping, elastic energy can be stored (during stance phase) in the muscle-tendon complex (or an ESR prosthesis) and it is released as mechanical work (during aerial phase). In this study spring effect of ESR is modelled using a trampoline.

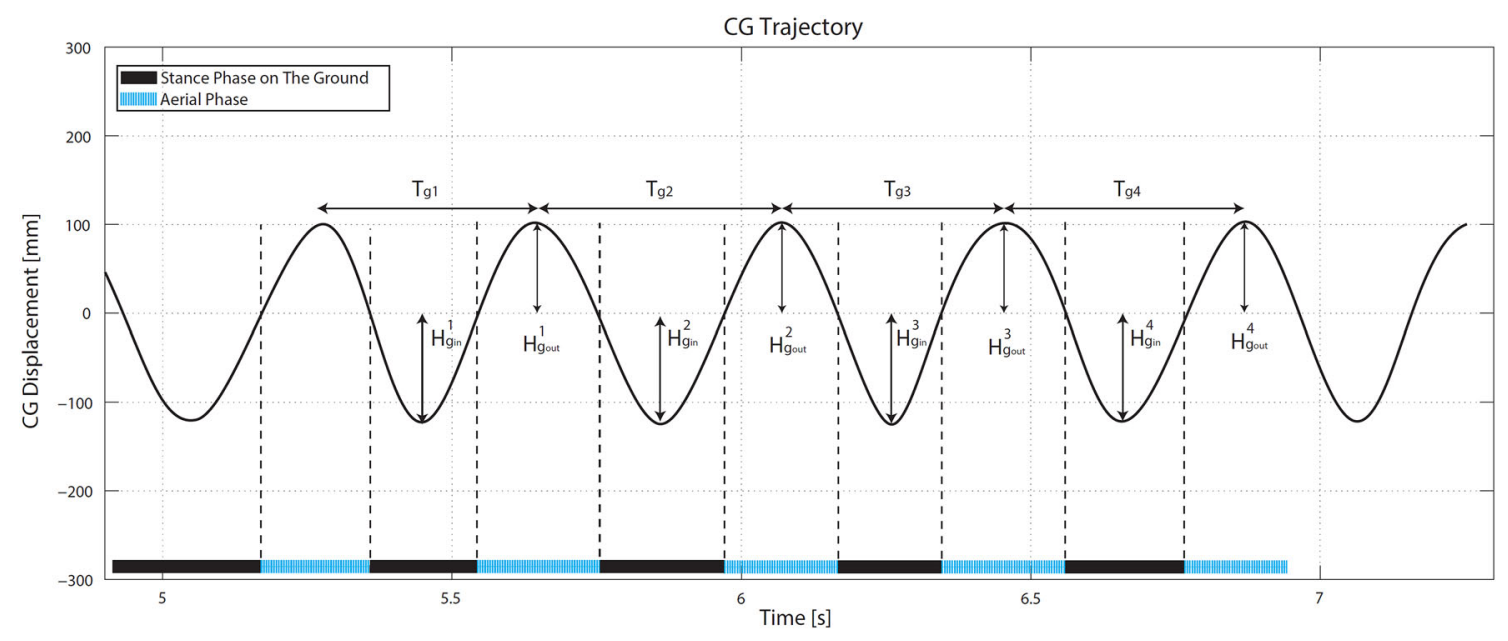

Fig. 3 Feet contacting the ground alternately 


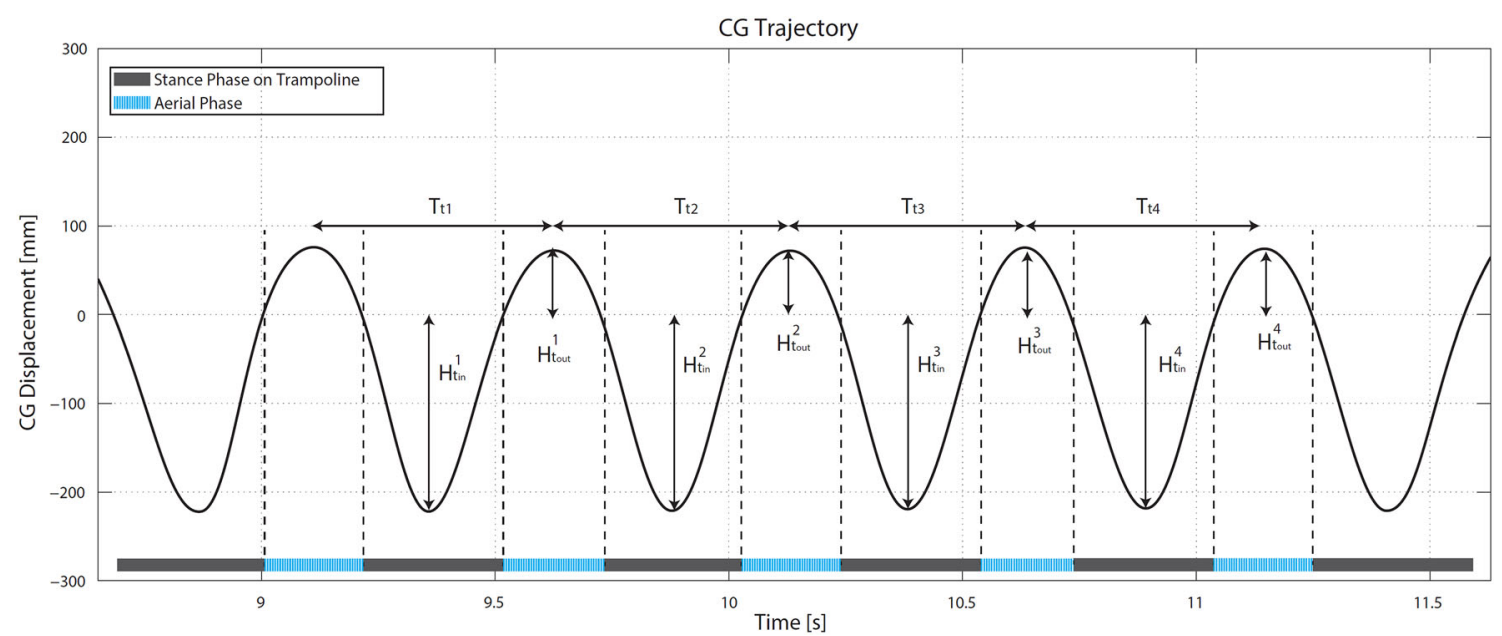

Fig. 4 Feet contacting the trampoline alternately

Table 1 Jumping frequency and energy transfer efficiency

\begin{tabular}{llll}
\hline Case number & 1 & 2 & 3 \\
\hline Average jumping frequency & $2.52 \mathrm{~Hz}$ & $1.95 \mathrm{~Hz}$ & $1.94 \mathrm{~Hz} / 2.43 \mathrm{~Hz}$ \\
Average energy transfer efficiency & $83 \%$ & $36 \%$ & $38 \% / 72 \%$ \\
\hline
\end{tabular}

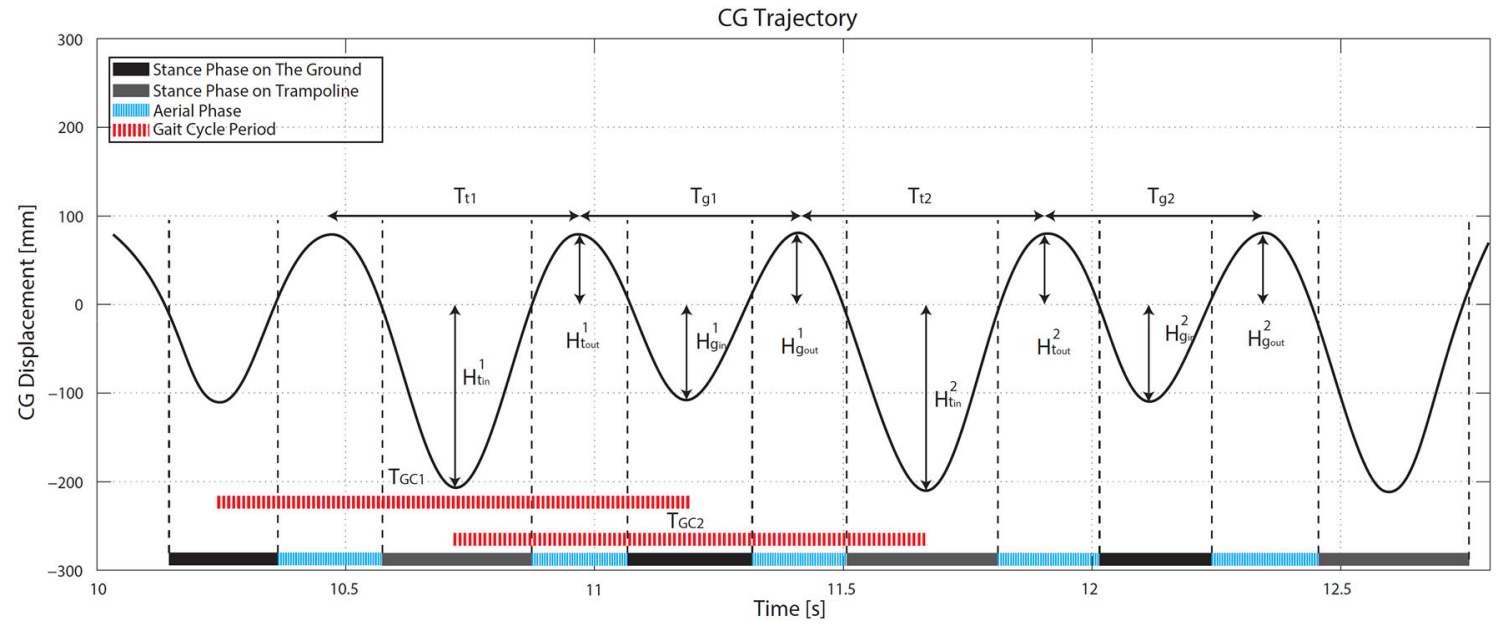

Fig. 5 One foot contacting the free half of trampoline and the other foot the constrained half

It is assumed that the CM displacement is proportional to the energy expenditure of the person when jumping according to previous studies [30-32]. Let the $H_{g_{\text {in }}}^{i}$ be the effective CM displacement that represents the input energy used by foot of a person touching the ground to achieve a certain height, and $H_{t_{i n}}^{i}$ to be effective CM displacement that represents the input energy used by foot of a person touching trampoline to achieve a certain height.

Energy transfer efficiency is defined as the ratio of stored elastic energy in muscle-tendon complex or ESR (represented by CM displacement during stance phase) to mechanical work represented by height of following jump
[33]. The energy return rate is evaluated as an aspect of energy consumption [34]. We can measure the energy output in term of CM displacement.

Depending on whether the foot is hitting the ground or trampoline, the jump energy transfer efficiency for $i$ th cycle, $\eta_{g}^{i}$ or $\eta_{t}^{i}$, can be found from:

$\eta_{g}^{i}=\frac{H_{g_{\text {out }}^{i}}^{i}}{H_{g_{\text {in }}}^{i}}, \eta_{t}^{i}=\frac{H_{t_{\text {out }}}^{i}}{H_{t_{\text {in }}}^{i}}$

where $H_{g_{\text {out }}}^{i}$ and $H_{t_{\text {out }}}^{i}$ are CM displacement at aerial phase (released mechanical work), $H_{g_{i n}}^{i}$ and $H_{t_{i n}}^{i}$ are $\mathrm{CM}$ displacement at stance phase (stored energy in muscle-tendon 
complex or ESR), and $g$ and $t$ indicate the ground and trampoline cases, respectively.

As shown in Table 1 in the first case average energy transfer efficiency is $83 \%$ while in the second case, efficiency decreases to $36 \%$. This reduction in energy return efficiency could be explained by the fact that a normal leg having functioning ankle joint is able to generate up to $241 \%$ energy return [1] while a leg using ESR (In this case trampoline) behaves as a mass (body weight) spring (ESR/trampoline) system to return stored energy and not generating any additional work.

In the third case one leg (contacting trampoline) experiences an average energy transfer efficiency of $38 \%$ while the other leg (touching the ground) has average energy transfer efficiency of $72 \%$ meaning in this case the subject needs to introduce excessive effort to compensate for asymmetry of jump efficiency between legs.

These results show that if both legs touch the ground, stored energy in the muscle-tendon complex and generated work by the ankle could produce greater mechanical work in comparison to the scenario which both legs experience the trampoline contact condition. It is also thought that if one leg contacts the ground while the other one contacts the trampoline, that the energy transfer efficiency between right and left leg will fluctuate which requires excessive compensation effort.

In this experiment a trampoline is used to simulate the ESR spring effect and it is concluded that unilateral runners require excessive effort to compensate for lack of symmetry as well as asymmetry in energy transfer, causing fatigue. Although trampoline and ESR have different dynamic elastic responses to impulse, both of them act as linear mass spring systems with fixed response characteristics which show limited range of output responses to variety of different inputs in different scenarios of motion.

\section{Conclusions}

An active ankle can both generate and return a large amount of mechanical energy (work) in a healthy leg, while the ESR foot, used by a below-knee amputee, can only store and return energy that is less than or at best equal to the potential energy stored in the body. The fixed nature of the mechanical characteristics of ESR feet causes limited output responses to a wide range of inputs. In a unilateral amputee this results in an unsymmetric gait which affects the gait efficiency compared to a healthy person or a bilateral amputee with symmetric gait. The dissimilar gait associated with different energy return between right and left in unilateral amputees is usually due to the excessive compensation needed in every step to control the divergence of the CM.
An ESR foot and mass system, resembling a linear mass spring one, generally has fixed response characteristics in terms of natural frequencies and their dynamic elastic response to an impulse, which is a function of both the initial condition and energy input into the system that can be stored in the mass using muscle power while running. Therefore for a given energy state or to maintain a steady energy state, muscle power needs to be applied at current amplitude and phase to store and return energy to the mass while the foot is in contact with the ground. The able foot, not being elastic, can generate different energy transfer efficiencies that can match that of the ESR foot but not vice versa. Hence a unilateral amputee must compensate for the lack of symmetry and try to match the prosthetic limb's elastic response in every step. This can result in in additional energy consumption and fatigue in the healthy leg and other limbs over longer distance running (over $100 \mathrm{~m}$ ), which can result in an unfair advantage of a bilateral amputee over a unilateral amputee.

A bilateral amputee wearing two similar ESR feet can have a symmetric gait. That in turn results in better energy return efficiency. The stored energy in a symmetric gait can later be recovered from the mass and help to sustain the momentum with little extra effort or be converted into higher velocity or more height allowing a large impulse.

In this paper the fixed characteristics of ESR feet are simulated by using a trampoline with fixed static and dynamic characteristics. Three different tests were conducted and it was found that in case of a simulated unilateral amputee maintaining symmetry was the main issue. Therefore it can be demonstrated that an able-bodied participant and a bilateral amputee can bounce symmetrically in jumping activity.

Technology is not yet ready to create a perfectly symmetric gait in unilateral amputees due to the long response time for sensing and the real-time stiffness adjustment system that are needed. The difference between the dynamics of unilateral and bilateral amputees when using ESR feet needs to be further investigated to help with fair classification of amputee athletes.

Open Access This article is distributed under the terms of the Creative Commons Attribution 4.0 International License (http://crea tivecommons.org/licenses/by/4.0/), which permits unrestricted use, distribution, and reproduction in any medium, provided you give appropriate credit to the original author(s) and the source, provide a link to the Creative Commons license, and indicate if changes were made.

\section{References}

1. Murnro C, Czerniecki JM, Gitter A (1991) Joint moment and muscle power output characteristic of below knee amputee during 
running: the influences of energy storing prosthetic feet. J Biomech 24(1):63-75

2. Nolan L (2008) Carbon fibre prostheses and running in amputees: a review. Foot Ankle Surg 14(3):125-129

3. Brüggemann G-P, Arampatzis A, Emrich F, Potthast W (2009) Biomechanics of double transtibial amputee sprinting using dedicated sprinting prostheses. Sport Technol 1(4-5):220-227

4. Noroozi S, Sewell P, Rahman AGA, Vinney J, Zhi Chao O, Dyer BTJ (2011) Performance enhancement of bi-lateral lower-limb amputees in the latter phases of running events: an initial investigation. 227(2):105-115

5. Hafner BJ, Sanders JE, Czerniecki JM, Fergason J (2002) Transtibial energy-storage-and-return prosthetic devices: a review of energy concepts and a proposed nomenclature. J Rehabil Res Dev 39(1):1-11

6. Buckley JG (1999) Sprint kinematics of athletes with lower-limb amputations. Arch Phys Med Rehabil 80(5):501-508

7. Weyand PG, Bundle MW, McGowan CP, Grabowski A, Brown MB, Kram R, Herr H (2009) The fastest runner on artificial legs: different limbs, similar function? J Appl Physiol 107(3):903-911

8. Hobara H, Baum BS, Kwon HJ, Shim JK (2015) Running mechanics in amputee runners using running-specific prostheses. no. April 2013, 2015

9. Grabowski AM, McGowan CP, McDermott WJ, Beale MT, Kram R, Herr HM (2010) Running-specific prostheses limit groundforce during sprinting. Biol Lett 6(2):201-204

10. Vinney J, Noroozi S, Rahman AGA, Sewell P, Chao OZ, Kuan KK, Dupac M (2012) Analysis of composite prosthetic energystoring-and-returning (ESR) feet: a comparison between FEA and the experimental analysis. Int J COMADEM 15(3):19-28

11. Noroozi S, Sewell P, Vinney J, Dyer BTJ, Rahman AGA, Zhi Chao O (2012) Modal analysis of composite prosthetic energystoring-and-returning feet: an initial investigation

12. Noroozi S, Rahman AG, Khoo SY, Zahedi S, Sewell P, Dyer B, Ong ZC (2013) The dynamic elastic response to impulse synchronisation of composite prosthetic energy storing and returning feet. Proc Inst Mech Eng Part P J Sport Eng Technol 228(1):24-32

13. Charlish P, Riley S (2008) Should oscar run?

14. Dyer BTJ, Noroozi S, Sewell P, Redwood S (2010) The design of lower-limb sports prostheses: fair inclusion in disability sport. August 2015, 2010

15. Hassani H, Ghodsi M, Shadi M, Noroozi S, Dyer B (2015) An Overview of the Running Performance of Athletes with LowerLimb Amputation at the Paralympic Games 2004-2012. Sports 3(2):103-115

16. Kaufman CA, Frittoli KR, Frigo S (2012) Gait asymmetry of transfemoral amputees using mechanical and microprocessorcontrolled prosthetic knees. Clin Biomech 27(5):460-465

17. Ganguli S, Mukherji P, Bose KS (1974) Gait evaluation of unilateral below-knee amputees fitted with patellar-tendon-bearing prostheses. J Indian Med Assoc 63(8):256-259
18. Hershler C, Milner M (1978) An optimality criterion for processing electromyographic (EMG) signals relating to human locomotion. IEEE Trans Biomed Eng 25(5):413-420

19. Arsenault AB, Winter DA, Marteniuk RG (1986) Bilateralism of EMG profiles in human locomotion. Am J Phys Med 65(1):1-16

20. Robinson RO, Herzog W, Nigg BM (1987) Use of force platform variables to quantify the effects of chiropractic manipulation on gait symmetry. J Manipulative Physiol Ther 10(4):172-176

21. Winter DA, Yack HJ (1987) EMG profiles during normal human walking: stride-to-stride and inter-subject variability. Electroencephalogr Clin Neurophysiol 67(5):402-411

22. Sadeghi H (2003) Local or global asymmetry in gait of people without impairments. Gait Posture 17(3):197-204

23. Haddad JM, Van Emmerik REA, Whittlesey SN, Hamill J (2006) Adaptations in interlimb and intralimb coordination to asymmetrical loading in human walking. Gait Posture 23(4):429-434

24. Zifchock RA, Davis I, Higginson J, Royer T (2008) The symmetry angle: a novel, robust method of quantifying asymmetry. Gait Posture 27(4):622-627

25. Petersen AO, Comins J, Alkjær T (2010) Assessment of gait symmetry in transfemoral amputees using C-Leg compared with 3R60 prosthetic knees. JPO J Prosthetics Orthot 22(2):106-112

26. Tura A, Raggi M, Rocchi L, Cutti AG, Chiari L (2010) Gait symmetry and regularity in transfemoral amputees assessed by trunk accelerations. J Neuroeng Rehabil 7(4):4

27. Dill DB (1965) Oxygen used in horizontal and grade walking and running on the treadmill. J Appl Physiol 20:19-22

28. Chang YH, Kram R (1999) Metabolic cost of generating horizontal forces during human running. J Appl Physiol 86(5):1657-1662

29. Gottschall JS, Kram R (2005) Energy cost and muscular activity required for leg swing during walking. J Appl Physiol 99(1):23-30

30. Hall C et al (2004) Energy expenditure of walking and running: comparison with prediction equations. Med Sci Sport Exerc 36(12):2128-2134

31. Rocha-Vieira E, Alves AS, Almeida LS, Pereira RL, Dickman AG, De Castro KM, Física CDE, De Física C, Universidade P, Gerais CDM, Horizonte B, Gerais M (2007) Energy cost during walking and running a same distance is associated with vertical oscillation on gravity center in Leonardo, 2007, pp. 248-251

32. Heglund NC, Cavagna GA, Willems PA (2000) The role of gravity in human walking: pendular energy exchange, external work and optimal speed. J Physiol 528(3):657-668

33. Kim S (2013) An effect of the elastic energy stored in the muscletendon complex at two different coupling-time conditions during vertical jump. Adv Phys Educ 03(01):10-14

34. Vanrenterghem J, Lees A, Lenoir M, Aerts P, De Clercq D (2004) Performing the vertical jump: movement adaptations for submaximal jumping. Hum Mov Sci 22(6):713-727 